\title{
Switzerland and the Digital Health Revolution
}

\author{
Didier Trono*
}

\begin{abstract}
New technologies in life sciences are helping to revolutionize health management with the objective to move towards personalized healthcare. The Swiss Federal government is investing in multidisciplinary, countrywide initiatives such as Health 2030 to meet this challenge.
\end{abstract}

Keywords: Personalized health

New technologies in life sciences, engineering, informatics and communication stand to revolutionize health management by opening the door to the establishment of connections between an individual's genotype (i.e. genetic make-up) and phenotype (i.e. the sum of traits that can be observed or measured), while integrating the influence of the environment (e.g. diet, exercise, exposure to chemicals, sunlight, viruses or bacteria). Medicine can thus ambition to morph from an essentially reactive and often empirical discipline into a precise, preventive, personalized and participatory endeavor. Individual risk factors will be identified to assess probability of developing diseases and design appropriate prophylactic measures, and ills such as cancer will be dealt with through highly specific and individualized treatments based on their precise molecular fingerprinting rather than by their assignment to overly general classes of pathologies.

For Switzerland, exploiting new technologies towards health management will require that the efforts of its biologists, chemists, bioengineers and medical doctors be complemented by the skills of its mathematicians, physicists and computer

${ }^{\star}$ Correspondence: Prof. Dr. D. Trono

EPFL

SV-GHI-LVG, Station 19

$\mathrm{CH}-1015$ Lausanne

E-mail: Didier.trono@epfl.ch scientists in order to process, store, protect, analyze and integrate the colossal sum of information that will be yielded not only by genomic, proteomic, metabolomics and metagenomic studies but also by epidemiological data of unprecedented diversity, to interpret the regulatory networks unveiled by these analyses, to develop and test complex models and theories, and to restitute this information not only to health care providers but to the entire population in a comprehensible and useful fashion. The success of this interdisciplinary enterprise will require a new generation of medical doctors endowed with truly quantitative and integrative skills, of life scientists and engineers sensitized to the complexity of human medicine, of computer scientists dedicated to solving the issues of medical data storage, encryption, and analysis, and of a whole range of other specialists geared to address the psychological, economical, ethical and legal issues linked to the exploitation of new technologies in the field of health. It will also require that this effort be conducted in continuous dialogue with society, in order to ensure the acceptability and maximize the efficiency of what should be a truly participatory process, secured by a legal framework that guarantees equal rights and social coverage to all citizens, irrespective of inter-individual differences that will be unveiled for instance by genetic data.

Switzerland has many assets for succeeding in this endeavor, including the quality and stability of its health care system, the education level of its population and its support for biomedicine, repetitively illustrated in the voting booths, the strength of its research in relevant sectors, its wealth, and its ability to react to societal challenges through the mobilization of stakeholders from all relevant constituencies. Yet the country needs to be swiftly and strongly reactive in developing, appropriating and mastering all the skills needed to ensure this success, in order to avoid that managing the health of its population be left instead in the hands of foreign internet or information technology companies that are motivated primarily by profit and are experts at escaping national laws.

Accordingly, Switzerland has launched major initiatives to meet this challenge. Personalized health is a line item in the 2017-2020 federal budget proposal currently examined by the Swiss parliament, and also a priority program of the Swiss Institutes of Technology domain for the same quadrennial period. If approved, the federal initiative will inject sizeable amounts of funds into the field, which will help build infrastructures, train specialists and run projects. Its general strategy will be defined and coordinated by a national steering board placed under the auspices of the Swiss Academy of Medical Sciences (http://www.samw.ch/fr/Projets/ Personalized-Health.html). Close collaboration between this body and both the Swiss National Science Foundation and the Federal Office of Public health (OFSP/ BAG) will be indispensable for a project that should encompass not only research and development in broad areas of science, engineering and the humanities, but also the rapid delivery to the general population of services based on duly validated new technologies.

This enterprise will also require the coordination of truly multi-disciplinary efforts that capitalize on the strengths of various parts of the country while overcoming the limitations of a system where the responsibility of health management is dispersed in the cantons. As a move towards this tricky exercise, the federal personalized health initiative will be organized around two clusters working in close collaboration, one grouping the forces of the Zurich-Basel region, the other those of Western Switzerland. This echoes the launching some time ago by the EPFL, the universities of Geneva and Lausanne and their affiliated hospitals of Health 2030, 
a project now joined by the university of Bern and its medical center. Health 2030 is a multi-centric, multidisciplinary initiative aimed at promoting research, education and services towards the advent of 'personalized medicine' through the exploitation of the new biomedical and information technologies by mobilizing all relevant parties, including primary care providers. It is organized around thematic networks, the broad coverage of which illustrates the holistic nature of this endeavor (Fig. 1).

Several challenges can be identified, which will need to be met for the success of personalized health in Switzerland:

1. Foster a 'Mannschaft spirit' at both national and regional levels, transgressing geographic and disciplinary barriers and overcoming institutional protectionism.

2. Carve optimal synergies between programs in personalized health and data science.

3. Properly define missions (e.g. digitalization of medical records, bioinformatics support, development of technolomic platforms) and funding channels (e.g. confederation vs. cantons, SNF vs. OFSP/BAG).

4. Ensure political support to personalized health, including through proper policy making.

5. Work towards societal acceptance of and broad community participation in the personalized health project.

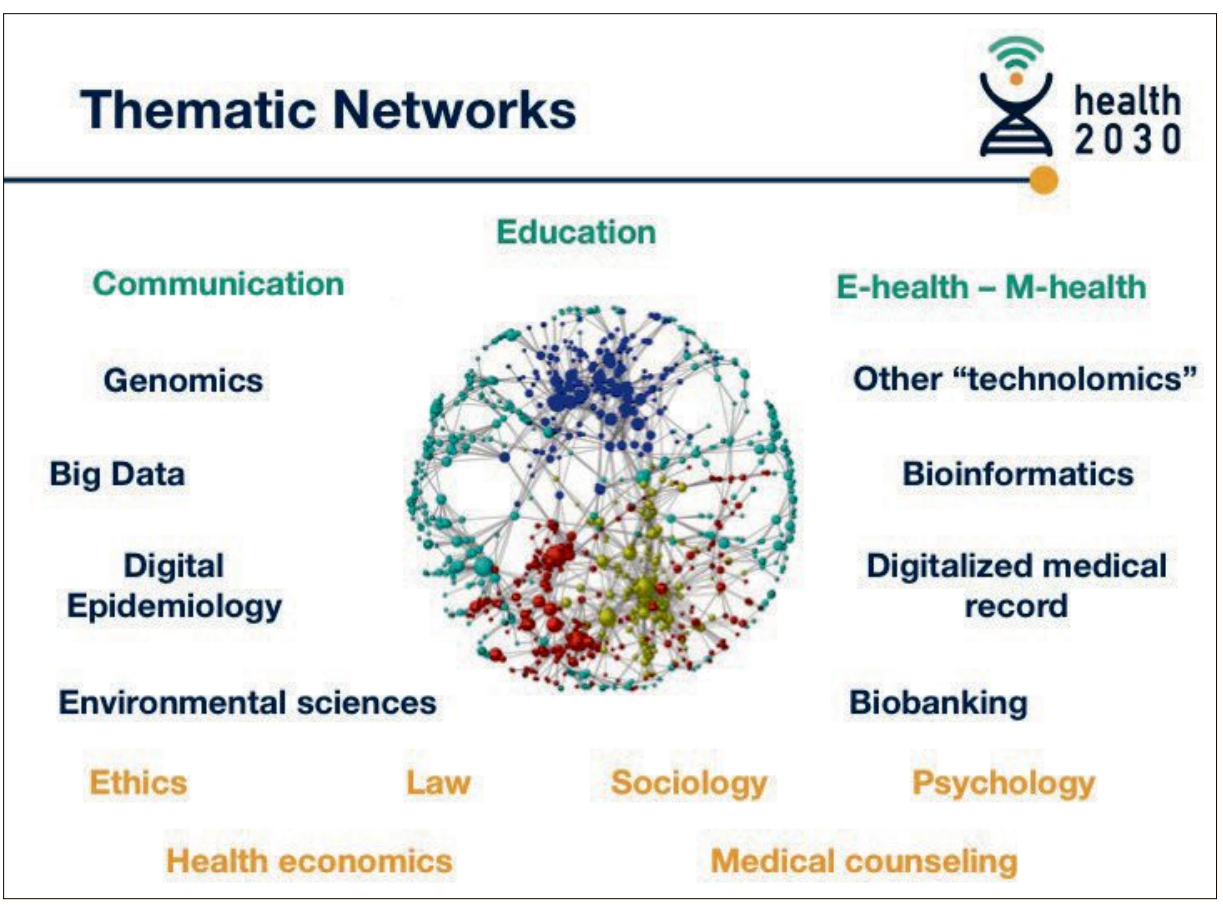

Fig. 1. Thematic networks contributing to the Health 2030 initiative. 